\title{
Hybrid esophagectomy for oesophageal cancer: long-term results. A single-centre experience
}

\author{
Lucie Hlaváčová ${ }^{1}$, Radek $\mathrm{Vrba}^{1}$, Čestmír Neoral ${ }^{1}$, René Aujeský${ }^{1}$, Martin Stašek $^{1}$, Josef Chudáček ${ }^{1}$, \\ Katherine Vomáčková ${ }^{1}$, David Vrána², Martin Sněhota ${ }^{3,4}$ \\ ${ }^{1}$ Department of Surgery I, University Hospital Olomouc, Faculty of Medicine and Dentistry, Palacký University, Olomouc, Czech Republic \\ ${ }^{2}$ Department of Oncology, AGEL Hospital, Nový Jičín, Czech Republic \\ ${ }^{3}$ Department of Medical Biophysics, Faculty of Medicine and Dentistry, Palacký University, Olomouc, Czech Republic \\ ${ }^{4}$ Centre for Telemedicine, Simulation and Clinical Skills, Faculty of Medicine and Dentistry, Palacký University, Olomouc, Czech Republic
}

Videosurgery Miniinv 2021; 16 (2): 297-304 DOI: https://doi.org/10.5114/wiitm.2020.100893

\begin{abstract}
Introduction: The authors report long-term outcomes in patients who received neoadjuvant chemoradiotherapy and consequently underwent hybrid oesophagectomy for oesophageal cancer (OC).

Aim: To evaluate long-term outcomes in patients suffering from OC, who underwent hybrid oesophagectomy. Material and methods: Our cohort consisted of patients suffering from OC, who received neoadjuvant chemoradiotherapy. Hybrid esophagectomy was performed 8-10 weeks after oncological treatment.

Results: Ninety-four patients underwent surgery for OC from 2011 to 2015. Histology revealed adenocarcinoma in $60.6 \%$, squamous cell carcinoma (SCC) in 36.2\%, and other type of cancer in $3.2 \%$. Seventy-three (77.7\%) patients with advanced stage (T3-4, NO-2, MO) were indicated to receive neoadjuvant chemoradiotherapy (nCRT). Trans-hiatal hybrid oesophagectomy was performed in 83 (88.3\%) patients. Transthoracic hybrid oesophagectomy was performed in 11 (11.7\%) patients. Histology of the resected specimens of 18 (24.7\%) patients did not reveal OC, i.e. pathological complete response ( $P C R)$. In our cohort, we proved an association between occurrence of $p C R$ and age as well as disease-free survival (DFS). The patients who presented with $p C R$ were significantly younger - below 60 years of age $(p=0.017)$. They also showed significantly higher mean DFS $(p=0.004)$.

Conclusions: Combined oesophagectomy with neoadjuvant chemoradiotherapy results in a better long-term outcome in patients suffering from oesophageal cancer. In our set of patients who underwent hybrid esophagectomy, satisfactory short-term and especially long-term results of surgical treatment for oesophageal cancer were observed.
\end{abstract}

Key words: oesophageal cancer, hybrid oesophagectomy, pathological complete response, long-term outcome.

\section{Introduction}

Oesophageal cancer (OC) is the seventh most common cancer diagnosed worldwide and the sixth most common cause of death among all cancers worldwide. A total of 572,034 new cases of OC $(3.2 \%$ of all cancers) were diagnosed in 2018. In the same year, 508,585 patients died from this disease $(5.3 \%$ of all deaths of cancer) [1]. Curative therapy of OC is radical oesophagectomy. Oesophagectomy is the removal of the oesophagus with the tumour along with lymphadenectomy according to oncological principles with subsequent reconstruction of the upper digestive tract utilising tubularised stomach or the large intestine. Mucosectomy is curative only for early stage of the disease (pT1a). Multimodal

\section{Address for correspondence}

Doc. MUDr. PhD. Radek Vrba, Department of Surgery I, University Hospital Olomouc, Faculty of Medicine and Dentistry,

Palacký University, Olomouc, Czech Republic, e-mail: radek.vrba@fnol.cz 
therapy in the form of neoadjuvant chemoradiotherapy (nCRT) is part of the therapeutic protocol for OC [2]. Even though therapy of OC has improved, the 5 -year survival rate after oesophagectomy ranges from $15 \%$ to $40 \%$ [3]. The main aim of this work is to evaluate long-term outcomes in patients suffering from OC, who underwent hybrid oesophagectomy. In addition, we also evaluated long-term survival in our set of patients with pathological compete response $(p C R)$ after multimodal therapy (neoadjuvant therapy, hybrid esophagectomy) in comparison with patients with partial response (nonCR). The retrospective study of the cohort of patients suffering from oesophageal cancer was approved by the ethics committee of University Hospital Olomouc.

\section{Aim}

The main aim of this work is to evaluate longterm outcome in patients suffering from OC, who underwent hybrid oesophagectomy. In addition, we evaluated long-term survival in our set of patients with pathological compete response (pCR) after multimodal therapy (neoadjuvant therapy, hybrid oesophagectomy) in comparison with patients with partial response (nonCR).

\section{Material and methods}

\section{Examination}

Oesophageal cancer was diagnosed based on endoscopic examination and tumour biopsy. Endosonography and PET/CT were used to determine TNM stage. Tracheobronchoscopy was performed in patients with tumours localised in the proximal and middle part of the oesophagus to rule out tumourous infiltration of the respiratory tract. All patients underwent general internal examination, spirometry, and nutritional evaluation before surgery. Based on the examinations, the patients were classified according to the ASA Physical Status Classification System (ASA).

\section{Neoadjuvant therapy}

Neoadjuvant therapy was indicated in cases of advanced clinical stage of the disease (T3-4, NO-2, $\mathrm{MO})$. In patients with tumourous stenosis of the oesophagus (revealed by endoscopy) and in patients suffering from significant dysphagia, a jejunostomy was performed to ensure nutrition prior to oncolog- ical therapy. Chemotherapy in the form of combination of 5 -fluoruracil and cisplatin was administered. The patients also received radiotherapy - total dose of 45-50.4 Gy and $1.8 \mathrm{~Gy} /$ fraction. The disease was restaged after chemoradiotherapy was finished (endoscopy and positron emission tomography/computed tomography (PET/CT) were performed) to assess the effect of neoadjuvant therapy. The patients underwent hybrid esophagectomy $8-10$ weeks after nCRT was completed.

\section{Surgery}

Oesophagectomy was performed using the hybrid approach, i.e. a combination of minimally invasive and classic open surgery. Resection was performed via minimally invasive approach, and reconstruction was performed by classic open surgery.

Transthoracic oesophagectomy was performed in cases of tumours localised in the thoracic part of the oesophagus. This approach was performed from a right thoracoscopy in modified prone position using selective intubation of the left bronchus with a collapsed right lung. Four incisions for $10 \mathrm{~mm}$ ports were made. The camera port was placed in the posterior axillary line in the fifth intercostal space, additional ports were placed in the scapular and mid-axillary line in the seventh intercostal space, and the last auxiliary port was located in the scapular line in the third intercostal space. The oesophagus with the tumour was dissected in its entire transthoracic course, and a harmonic scalpel or LigaSure was used to dissect the mediastinal pleura. The azygos vein was transected by vascular endostapler or with the use of locking Endoclips. Total mediastinal lymphadenectomy was performed during thoracoscopy.

Minimally invasive trans-hiatal laparoscopy was performed in cases of tumours localised in the distal part of the oesophagus and gastroesophageal junction. In this approach the patients were in the supine position with abducted legs and operated under endotracheal anaesthesia. The operation was performed using five incisions with five ports; four $10 \mathrm{~mm}$ ports and one $5 \mathrm{~mm}$ port. The laparoscope with $30^{\circ}$ optics was introduced into the port located $5 \mathrm{~cm}$ above umbilicus. Standard mediastinal lymphadenectomy was also performed.

Reconstruction was performed after the transthoracic or trans-hiatal resection phase. We used a left-sided cervical incision in both cases. The oesoph- 
agus was completely transected in the deep cervical space. Consequently, the oesophagus with tumour was removed via minilaparotomy. Tubularised gastric conduit was used for transposition in all patients. To construct the gastroplasty, the small curvature of the stomach and the oesophagus were resected using linear staplers; the resection line was always reinforced by suture. The right epiploic artery was preserved to provide nutrition to the conduit. Anastomosis of the gastroplasty and cervical part of the oesophagus was performed using a single-layer continuous suture. Pyloroplasty was also performed in all cases.

The patients were transferred to the intensive care unit after the surgery. Nutrition was administered enterally (nasojejunal probe) and in parenteral form. An X-ray swallow test was performed on day 7 after the surgery. In the case of a satisfactory result and absence of anastomotic fistula, the patients were slowly transferred back to classic enteral nutrition. The patients were followed-up every 3 months for the next 3 years. Follow-up abdominal ultrasonography and chest X-ray were performed every 6 months. PET/CT was performed if necessary. The patients were then followed-up every 6 months during the subsequent 2 years. Follow-up abdominal ultrasonography and chest X-ray were performed once a year.

\section{Statistical analysis}

IBM SPSS Statistics version 22 was used for data analysis. Qualitative parameters of cohorts were compared using the $\chi^{2}$ test and Fisher's exact test, respectively. Student's $t$-test was used for comparison of ages. Kaplan-Meier curve was used for comparison of overall survival (OS) and disease-free survival (DFS) (Figure 1). All tests were performed at the level of significance 0.05 .

\section{Results}

Ninety-four patients (81 males - $86.2 \%$ and 13 females - $13.8 \%$ ) underwent surgery for OC from 2011 to 2015 (Table I). The average age of our cohort was 63.9 years (the youngest patient was 34 years old and the oldest was 81 years old). Thirty-eight (40.4\%) patients were younger than 60 years, and the remaining $56(59.6 \%)$ patients were older than 60 years. Adenocarcinoma was present in 57 (60.6\%) patients, squamous cell carcinoma (SCC) was present in $34(36.2 \%)$ patients, and other tumour type (small- cell, mucinous carcinoma) was present in 3 (3.2\%) patients. In 34 (36.2\%) patients the tumour was localised in the upper or middle part of the oesophagus. Sixty (63.8\%) patients had the tumour localised in the lower part of the oesophagus or in the gastroesophageal junction. Twenty-one (22.3\%) patients were indicated for primary surgery based on TNM classification (T1-2NOM0). Seventy-three (77.7\%) patients were indicated for nCRT due to more advanced stage of the disease (T3-4, N0-2, MO). The patients were classified according to American Society of Anaesthesiologists Classification (ASA Class). Forty-nine (52.1\%) patients were classified as ASA 3, 43 (45.7\%) patients were classified as ASA 2, and 2 (2.1\%) patients were classified as ASA 1. Trans-hiatal hybrid oesophagectomy was performed in 83 (88.3\%) patients. Transthoracic hybrid oesophagectomy was performed in 11 (11.7\%) patients. Trans-hiatal oesophagectomy during the resection phase never required conversion to classic open surgery. Transthoracic minimally invasive approach had to be converted to classic open right thoracotomy due to the size of the tumour and its close anatomical relation to the tracheal bifurcation in 3 cases. Severe intraoperative complications did not occur.

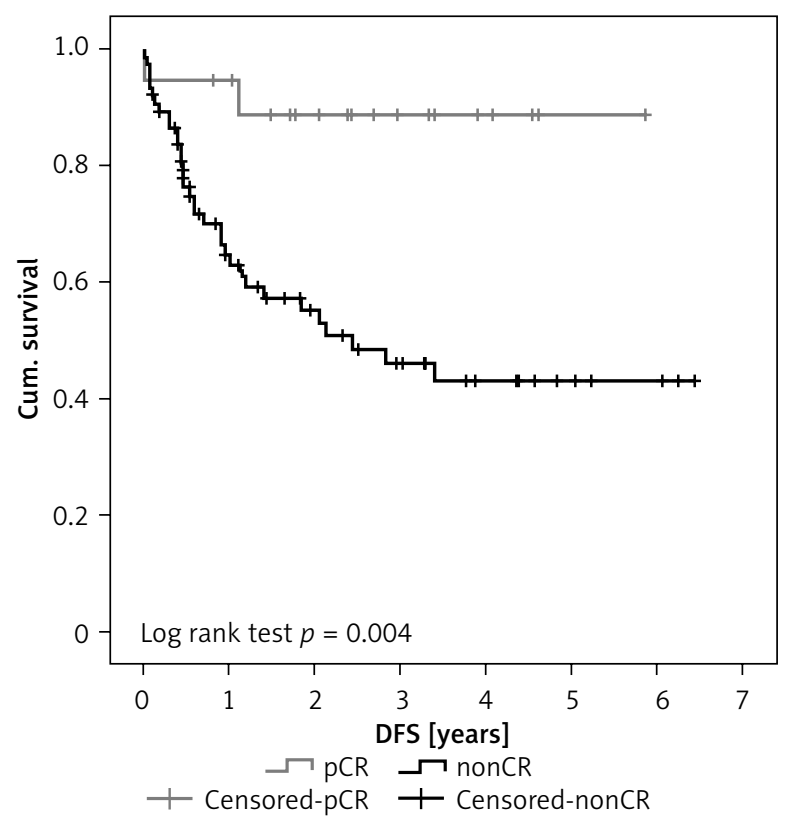

Figure 1. Kaplan-Meier curve of DFS of patients with $\mathrm{pCR}$ and nonCR. Probability of DFS of 4 years or more is approximately 0.9 in patients with pCR. On the other hand, DFS of 4 years and more is approximately 0.4 in nonCR patients 
Blood loss ranged from $100 \mathrm{ml}$ to $3500 \mathrm{ml}$, with an average of $555 \mathrm{ml}$. Blood transfusion had to be administered to 52 (55.3\%) patients either during or after the surgery. Duration of surgery ranged from

Table I. Cohort of patients who underwent hybrid oesophagectomy for oesophageal cancer from 2011 to 2015

\begin{tabular}{|c|c|c|}
\hline Parameter & Count & $\%$ \\
\hline $\begin{array}{l}\text { Total number of patients with } \\
\text { oesophageal cancer }\end{array}$ & 94 & 100 \\
\hline Males/females & $81 / 13$ & $86.2 / 13.8$ \\
\hline $\begin{array}{l}\text { Age below } 60 \text { years/Age above } \\
60 \text { years }\end{array}$ & $38 / 56$ & $40.4 / 59.6$ \\
\hline ASA class I, II, III & $2,43,49$ & $2.1 / 45.7 / 52.1$ \\
\hline Squamous cell carcinoma & 34 & 36.2 \\
\hline Adenocarcinoma & 57 & 60.6 \\
\hline Other type of tumour & 3 & 3.2 \\
\hline Neoadjuvant therapy & 73 & 77.7 \\
\hline $\begin{array}{l}\text { Hybrid trans-hiatal oesophagec- } \\
\text { tomy }\end{array}$ & 83 & 88.3 \\
\hline $\begin{array}{l}\text { Hybrid transthoracic oesophagec- } \\
\text { tomy }\end{array}$ & 11 & 11.7 \\
\hline Blood transfusion & 52 & 55.3 \\
\hline $\begin{array}{l}\text { Total number of respiratory com- } \\
\text { plications }\end{array}$ & 46 & 48.9 \\
\hline $\begin{array}{l}\text { Severe respiratory complications } \\
\text { (ARDS, respiratory failure) }\end{array}$ & 11 & 11.7 \\
\hline $\begin{array}{l}\text { Mild respiratory complications } \\
\text { (pneumonia, fluidothorax, atelec- } \\
\text { tasis, pneumothorax) }\end{array}$ & 35 & 37.2 \\
\hline Cardiac complications & 8 & 8.5 \\
\hline Anastomotic fistula & 9 & 9.6 \\
\hline 30-day mortality & 2 & 2.1 \\
\hline 90-day mortality & 2 & 2.1 \\
\hline \multicolumn{3}{|l|}{ The Clavien-Dindo classification: } \\
\hline Grade I & 10 & 10.3 \\
\hline Grade II & 47 & 50 \\
\hline Grade IIIa & 17 & 18 \\
\hline Grade IIIb & 7 & 7.4 \\
\hline Grade IVa & 5 & 5.3 \\
\hline Grade IVb & 1 & 1 \\
\hline Grade V & 4 & 4.2 \\
\hline
\end{tabular}

$130 \mathrm{~min}$ to $345 \mathrm{~min}$, with an average of $222 \mathrm{~min}$. Lymphadenectomy was performed in all patients. On average, 11.7 lymph nodes were removed (minimum 5 and maximum 28). Respiratory complications predominated in the postoperative period. Severe respiratory complications (acute respiratory distress syndrome (ARDS) and respiratory failure) occurred in $11(11.7 \%)$ patients. Mild respiratory complications (pneumonia, pleural effusion, postoperative pneumothorax, and atelectasis) occurred in 35 (37.2\%) patients. Cardiac complications occurred in $8(8.5 \%)$ patients. Anastomotic fistula developed in 9 (9.6\%) patients. Three fistulas required a combination of surgical drainage of the deep cervical space and antibiotic therapy. Six fistulas were managed conservatively - the fistula was small in all cases and follow-up swallow test was physiological. Paresis of the recurrent laryngeal nerve developed in $9(9.6 \%)$ patients. Oedema in the region of surgery was the cause in 5 cases. The pareses improved after some time. An incisional hernia developed in $3(3.1 \%)$ patients. It was treated surgically by mesh implantation. The shortest hospitalisation lasted 11 days and the longest was 119 days, with an average of 22.5 days.

Two patients died during the 30-day postoperative period due to severe respiratory complications, and 2 patients died during the 90-day postoperative period due to respiratory complications and multi-organ dysfunction syndrome that developed due to sepsis resulting from massive dehiscence of the anastomosis and mediastinitis. The complications were classified according to the Clavien-Dindo Classification - 10 (10.6\%) patients showed grade I, 47 (50\%) patients showed grade II, 17 (18\%) patients showed grade IIla, 7 (7.4\%) patients showed grade IIIb, 5 (5.3\%) patients showed grade IVa, 1 (1\%) patient showed grade IVb, and 4 (4.2\%) patients showed grade $\mathrm{V}$.

Eighteen $(24.7 \%)$ patients presented with pathological complete response (pCR) to neoadjuvant therapy in the resected specimen, i.e. no tumour was present. The remaining 55 (75.3\%) patients presented with signs of malignant tumour in the resected specimen (nonCR). Statistical analysis of our results revealed the following: We proved a statistically significant association between $\mathrm{pCR}$ and age. Patients with $P C R$ were significantly younger - they were mostly below 60 years of age $(p=0.017)$, Table II. Patients with $\mathrm{PCR}$ also showed significantly longer DFS, 3.4 years vs. 2.5 years $(p=0.004)$, Figure 1 . 
Table II. Comparison of patients with $p C R$ and without $p C R$. Patients with $p C R$ were significantly younger - below 60 years of age $(p=0.017)$. Statistically significant difference in sex, tumour type, its localisation, and the number of deaths was not proven

\begin{tabular}{|c|c|c|c|c|c|}
\hline \multirow[t]{2}{*}{ Parameter } & \multicolumn{2}{|c|}{ nonCR } & \multicolumn{2}{|c|}{$\mathrm{pCR}$} & \multirow[t]{2}{*}{$P$-value } \\
\hline & Count & $\%$ & Count & $\%$ & \\
\hline \multicolumn{5}{|l|}{ Sex: } & 0.721 \\
\hline Male & 46 & 83.6 & 16 & 88.9 & \\
\hline Female & 9 & 16.4 & 2 & 11.1 & \\
\hline \multicolumn{5}{|l|}{ Age: } & 0.017 \\
\hline Below 60 years & 19 & 34.5 & 12 & 66.7 & \\
\hline Above 60 years & 36 & 65.5 & 6 & 33.3 & \\
\hline \multicolumn{5}{|l|}{ Tumour type: } & 0.509 \\
\hline Adenocarcinoma & 31 & 56.4 & 8 & 44.4 & \\
\hline Squamous cell carcinoma & 22 & 40.0 & 10 & 55.6 & \\
\hline Other & 2 & 3.6 & 0 & 0.0 & \\
\hline \multicolumn{5}{|l|}{ Tumour localisation: } & 1.000 \\
\hline Upper and middle & 23 & 41.8 & 6 & 33.3 & \\
\hline Distal & 32 & 58.2 & 12 & 66.7 & \\
\hline \multicolumn{5}{|l|}{ Death: } & 0.523 \\
\hline No & 51 & 92.7 & 17 & 94.4 & \\
\hline Yes & 4 & 7.3 & 1 & 5.6 & \\
\hline
\end{tabular}

A statistically significant difference was not proven for different sex, tumour type, tumour localisation, and OS after the surgery.

Patients with adenocarcinoma had the tumour significantly more often localised in the distal part of the oesophagus $(p<0.0001)$, Table III. A statistically significant difference was not proven for different sex, pCR, OS, and DFS. Men and women did not show a statistically significant difference in tumour type, localisation, $\mathrm{PCR}$, and number of deaths. Women were significantly older than men $(p=0.041)$. No statistically significant relationship between sex and OS $(p=0.135)$ or DFS $(p=0.616)$ was proven.

\section{Discussion}

The only curative therapy for $\mathrm{OC}$ is oesophagectomy. It can be performed using classic open surgery, hybrid technique (combination of classic open surgery and minimally invasive approach), or completely using the minimally invasive approach. The choice of a particular approach should be based on preoperative staging of the disease, the size of the tumour, its histological characteristics, the patient's comorbidities, the anatomical and physiological status of the patient, and the experience of the surgeon [4]. Minimally invasive techniques have been preferred over the past decade. Less surgical trauma, fewer respiratory complications, and shorter recovery period are reported in cases of minimally invasive oesophagectomy [5, 6]. Hybrid oesophagectomy is also associated with better postoperative outcomes compared to the open approach, especially concerning pulmonary complications. For long-terms outcomes, the hybrid approach showed similar, or even better, overall survival than the open approach [7].

It is important to preserve surgical radicality with proper lymphadenectomy when using any of the approaches [8]. Tachibana et al. recommend removing at least 12 lymph nodes [9]. The number of metastatic lymph nodes ( $<3$ vs. $\geq 3$ ) was an independent prognostic factor for OS ( $p=0.02)$, as demonstrated by Gulben et al.; the 5-year OS rates in patients with < 3 MINs and $\geq 3$ MINs were 17\% (median: 34 months) and 5\% (median: 18 months), respectively [10]. 
Table III. Comparison of results according to tumour type. Patients suffering from adenocarcinoma showed significantly higher occurrence of tumour in the distal part of the oesophagus $(p<0.0001)$. Statistically significant difference in sex, age, presence of $\mathrm{pCR}$, and number of deaths was not proven

\begin{tabular}{|c|c|c|c|c|c|}
\hline \multirow[t]{3}{*}{ Parameter } & \multicolumn{4}{|c|}{ Tumour type } & \multirow[t]{3}{*}{$P$-value } \\
\hline & \multicolumn{2}{|c|}{ Adenoca } & \multicolumn{2}{|c|}{ Squamous cell carcinoma } & \\
\hline & Count & $\%$ & Count & $\%$ & \\
\hline \multicolumn{5}{|l|}{ Sex: } & 0.543 \\
\hline Male & 50 & 87.7 & 28 & 82.4 & \\
\hline Female & 7 & 12.3 & 6 & 17.6 & \\
\hline \multicolumn{5}{|l|}{ Age: } & 0.193 \\
\hline Below 60 years & 19 & 33.3 & 16 & 47.1 & \\
\hline Above 60 years & 38 & 66.7 & 18 & 52.9 & \\
\hline \multicolumn{5}{|l|}{ Tumour localisation: } & $<0.0001$ \\
\hline Upper and middle & 7 & 12.3 & 26 & 76.5 & \\
\hline Distal & 50 & 87.7 & 8 & 23.5 & \\
\hline \multicolumn{5}{|l|}{ pCR: } & 0.122 \\
\hline No & 48 & 84.2 & 24 & 70.6 & \\
\hline Yes & 9 & 15.8 & 10 & 29.4 & \\
\hline \multicolumn{5}{|l|}{ Death: } & 0.749 \\
\hline No & 15 & 26.3 & 10 & 29.4 & \\
\hline Yes & 42 & 73.7 & 24 & 70.6 & \\
\hline
\end{tabular}

A multicentric randomised controlled trial (total number of patients 207) showed that the use of hybrid minimally invasive oesophagectomy results in a decrease of severe intraoperative and postoperative complications (especially respiratory) by $77 \%$ when compared to classic open oesophagectomy. Overall survival and disease-free survival were the same or longer in patients after minimally invasive surgery compared to patients having undergone the open procedure over a period of 3 years [11]. We prefer the hybrid oesophagectomy at our workplace. Resection is performed by minimally invasive approach, and reconstruction is done via classic open surgery. The surgery is performed via trans-hiatal approach for tumours located in the aboral part of the oesophagus. Thus, incision of the thoracic wall is not necessary. Reconstruction is performed using the tubularised gastric conduit. Anastomosis to the remaining cervical oesophagus is then performed in the deep cervical space. Coloplasty is performed when the stomach cannot be used as transponate (due to malignant infiltration of oesophagus or previous gastric surgery).

To achieve optimal short- and long-term results following surgery for oesophageal cancer, oesophageal surgeons are in agreement that these procedures should be performed in specialised high-volume centres. Minimally invasive procedures should be performed by experienced surgeons in centres specialising in minimally invasive procedures.

In the past, oesophagectomy was associated with a high perioperative morbidity and mortality. Nowadays, perioperative morbidity ranges from $20 \%$ to $50 \%$ [12, 13]. Respiratory complications are most serious and occur in $19-44 \%$ of all cases [14]. These are dangerous especially for old patients, smokers, patients suffering from malnutrition, patients with low forced expiratory volume in $1 \mathrm{~s}\left(\mathrm{FEV}_{1}\right)$, patients who have received neoadjuvant therapy, and patients who develop anastomotic fistula [12, 13]. Our cohort of patients also experienced respiratory complications to be most com- 
mon, which corresponds with the experience of other research teams. Respiratory complications developed in $48.9 \%$ of cases $(11.7 \%$ were severe and $37.2 \%$ were mild). Necrosis of the transponate is most dangerous from a surgical point of view. However, this complication is rare $-0.5 \%$ [15]. According to the literature, dehiscence of the anastomosis occurs in $5-20 \%$ of all cases [16].

Perioperative mortality is defined as death during surgery or in the 30 following days. Postoperative mortality significantly decreased when compared to previous decades, especially in high-volume centres where it ranges from $1 \%$ to $5 \%$ [17, 18]. A study of the Society of Thoracic Surgeons General Thoracic Surgery Database (STS GTSD) reported perioperative mortality of $3.1 \%$ in a cohort of 4321 patients who underwent oesophagectomy [19]. Also, a study of American College of Surgeons National Surgical Quality Improvement Program (ACS-NSQIP) which analysed a cohort of 1032 patients who underwent oesophagectomy reported a 3.0\% mortality [20].

Even though treatment of oesophageal tumours has improved, the 5-year survival rate still ranges from $15 \%$ to $40 \%$ [3]. The randomised study Chemo Radiotherapy for Oesophageal cancer followed by Surgery Study (CROSS) reported an increase in 5-year survival rate by $14 \%$ in patients who received nCRT with consequent surgery when compared to patients who underwent only surgery. Moreover, $29 \%$ of patients who received nCRT showed pCR $(49 \%$ of SCC and $23 \%$ of adenocarcinoma) [21]. Similar results were observed in our study; nearly $25 \%$ of our patients had pCR in the definite histological finding.

Chirieac et al. studied a cohort of 235 patients either with invasive SCC or with adenocarcinoma of the oesophagus or oesophagogastric junction. Residual carcinoma in the resected specimen was divided into four categories: no residual carcinoma (stage 0), $1-10 \%$ of residual carcinoma (stage I), $11-50 \%$ of residual carcinoma (stage $\mathrm{II}$ ), and $>50 \%$ of residual carcinoma (stage III). The DFS rate was significantly higher in patients with stage 0 (60\% after 5 years) when compared to patients with more advanced stages. Patients with stage I and stage II did not show a statistically significant difference in DFS $(p=0.67)$. The OS rate of patients in stage 0 was $65 \%$ after 5 years. Patients in other stages with residual carcinoma showed an OS rate of $29 \%(p=0.003)$ after 5 years. Chirieac also points out that preoperative results of examinations as well as clinical stage of the disease did not have any influence on DFS and OS. He better predicts the OS based on findings of residual carcinoma in the resected oesophagus or gastroesophageal junction. The patients who presented with no residual carcinoma in the resected specimen showed significantly better results [22]. In our cohort we also had patients who showed pathological complete response to neoadjuvant chemoradiotherapy, i.e. no residual carcinoma was revealed in the resected specimen. Pathological complete response was present in $24.7 \%$ of cases. There was no statistically significant difference in occurrence of pCR in patients with adenocarcinoma and SCC.

Darton et al. and Meluch et al. also report similar results [23, 24]. Lin et al. showed in their study that $\mathrm{PCR}$ is a positive prognostic factor of better outcome in patients with locally advanced tumour, who received $\mathrm{nCRT}$ and consequently underwent oesophagectomy [25]. Alnaji et al. analysed a cohort of patients with oesophageal adenocarcinoma, who received $\mathrm{nCRT}$ and consequently underwent oesophagectomy. Nineteen percent of patients showed pathological complete response. Overall 3-year survival was $86 \%$ in patients who showed pCR. On the other hand, overall 3-year survival of patients who showed nonCR was $48 \%$. The disease-free survival rate was $80 \%$ in patients with $\mathrm{pCR}$ and $39 \%$ in patients with nonCR. Thus, pCR can be considered as an independent prognostic factor of better OS and DFS [26]. In our cohort we proved significantly longer DFS when compared to the aforementioned studies. However, we did not prove longer OS.

\section{Conclusions}

Combined oesophagectomy with neoadjuvant chemoradiotherapy results in better long-term outcome in patients suffering from oesophageal cancer. In our set of patients, who underwent hybrid oesophagectomy, satisfactory short-term and especially long-term results of surgical treatment for oesophageal cancer were observed. Further studies are necessary to support the results reported in this manuscript.

\section{Acknowledgments}

The study was supported by Ministry of Health, Czech Republic - conceptual development of research organization (FNOL, 00098892). 


\section{Conflict of interest}

\section{The authors declare no conflict of interest.}

\section{References}

1. GLOBOCAN 2018: Estimated cancer incidence, mortality and prevalence worldwide in 2018. Available from: http://gco.iarc.fr/ today/data/factsheets/cancers/6-Oesophagus-fact-sheet.pdf.

2. Ninomiya I, Osugi H, Fujimura T, et al. Thoracoscopic esophagectomy with extended lymph node dissection in the left lateral position: technical feasibility and oncologic outcomes. Dis Esophagus 2014; 27: 159-67.

3. Huang FL, Yu SJ. Esophageal cancer: Risk factors, genetic association, and treatment. Asian J Surg 2018; 41: 210-5.

4. Bonavina L, Asti E, Sironi A, et al. Hybrid and total minimally invasive esophagectomy: how I do it. J Thorac Dis 2017; 9 (Suppl 8): S761-72.

5. Meng F, Li Y, Ma H, et al. Comparison of outcomes of open and minimally invasive esophagectomy in 183 patients with cancer. J Thorac Dis 2014; 6: 1218-24.

6. Vrba R, Vrána D, Neoral Č, et al. Respiratory complications following mini-invasive laparoscopic and thoracoscopic esophagectomy for esophageal cancer. Experience in $215 \mathrm{pa}$ tients. Videosurgery Miniinv 2019; 14: 52-9.

7. Thibault V, Alexandru L, Guillaume P. Hybrid esophagectomy. J Thorac Dis 2019; 11 (Suppl 5): S723-7.

8. Vrba R, Aujeský R, Stašek M, et al. Esophageal cancer results of surgical treatment at the Department of Surgery I at the University Hospital Olomouc. Rozhl Chir Winter 2018; 97: 342-8.

9. Tachibana M, Kinugasa S, Hirahara N, et al. Lymph node classification of esophageal squamous cell carcinoma and adenocarcinoma. Eur J Cardiothorac Surg 2008; 34: 427-31.

10. Gulben K, Irkin F, Yazı M, et al. Prognostic significance of number of lymph node metastasis on survival in patients with pathological T3 esophageal carcinoma. Neoplasma 2017; 64: 131-5.

11. Mariette C, Markar SR, Dabakuyo-Yonli TS, et al. Hybrid minimally invasive esophagectomy for esophageal cancer. N Engl J Med 2019; 380: 152-62.

12. Wlodarczyk J, Kużdżał J. Stenting as a palliative method in the management of advanced squamous cell carcinoma of the oesophagus and gastro-oesophageal junction. Videosurgery Miniinv 2016; 11: 1-8.

13. Zhang Z, Zhang H. Impact of neoadjuvant chemotherapy and chemoradiotherapy on postoperative cardiopulmonary complications in patiens with esophagea lcancer. Dis Esophagus 2017; 30: 1-7.

14. Ott K, Bader FG, Lordick F, et al. Surgical factors influence the outcome after Ivor-Lewis esophagectomy with intrathoracic anastomosis for adenocarcinoma of the esophagogastric junction: a consecutive series of 240 patient at an experienced center. Ann Surg Oncol 2009; 16: 1017-25.

15. Wormuth JK, Heitmiler RF. Esophageal conduit necrosis. J Thorac Surg Clin 2006; 16: 11-22.

16. Haga Y, Wada Y, Takeuchi H, et al. Prediction of anasto-motic leak and its prognosis in digestive surgery. World J Surg 2011; 35: 716-22.
17. Atkins BZ, Shah AS, Hutcheson KA, et al. Reducing hospital morbidity and mortality following esophagectomy. Ann Thorac Surg 2004; 78: 1170-6.

18. Xing XZ, Gao Y, Wang HJ, et al. Assessment of a predictive score for pulmonary complications in cancer patients after esophagectomy. World J Emerg Med 2016; 7: 44-9.

19. Raymond DP, Seder CW, Wright CD, et al. Predictors of major morbidity or mortality after resection for esophageal cancer: a Society of Thoracic Surgeons General Thoracic Surgery Database Risk Ajustment Model. Ann Thorac Surg 2016; 102: 207-14.

20. Dhungel B, Diggs BS, Hunter JG, et al. Patient and peri-operative predictors of morbidity and mortality after esophagectomy: American College of Surgeons National Surgical Quality Improvement Program (ACS-NSQIP), 2005-2008. J Gastrointest Surg 2010; 14: 1492-501.

21. van Hagen P, Hulshof MC, van Lanschot JJ, et al. Preoperative chemoradiotherapy for esophageal or junctional cancer. N Engl J Med 2012; 366: 2074-84.

22. Chirieac LR, Swisher SG, Ajani JA, et al. Posttherapy pathologic stage predicts survival in patients with esophageal carcinoma receiving preoperative chemoradiation. Cancer 2005; 103 : 1347-55.

23. Darnton SJ, Archer VR, Stocken DD, et al. Preoperative mitomycin, ifosfamide, and cisplatin followed by esophagectomy in squamous cell carcinoma of the esophagus: pathologic complete response induced by chemotherapy leads to long-term survival. J Clin Oncol 2003; 21: 4009-15.

24. Meluch AA, Greco FA, Gray JR, et al. Preoperative therapy with concurrent paclitaxel/carboplatin/infusional 5-FU and radiation therapy in locoregional esophageal cancer: final results of a Minnie Pearl Cancer Research Network phase II trial. Cancer J 2003; 9: 251-60.

25. Lin JW, Hsu CP, Yeh HL, et al. The impact of pathological complete response after neoadjuvant chemoradiotherapy in locally advanced squamous cell carcinoma of esophagus. J Chin Med Assoc 2018; 81: 18-24.

26. Alnaji RM, Du W, Gabriel E, et al. Pathologic complete response is an independent predictor of improved survival following neoadjuvant chemoradiation for esophageal adenocarcinoma. J Gastrointest Surg 2016; 20: 1541-6.

Received: 24.09.2020, accepted: 12.10.2020. 